\title{
6 Limitationen der Studie
}

Die internationalen Entwicklungen innerhalb des univentrikulären Patientenkollektivs wurden anhand einer Übersicht der bislang veröffentlichten Literatur untersucht. Durch die begrenzte Anzahl der miteinbezogenen Studien und deren sehr unterschiedliche Protokolle konnten hier nur die Trends demonstriert werden.

Für die Untersuchung des Langzeitverlaufs der adoleszenten und adulten Patienten wurden die Ergebnisse der postoperativen Routineuntersuchungen retrospektiv ausgewertet. Durch die aus epidemiologischer Sicht relativ geringe Patientenanzahl war die statistische Auswertbarkeit nur eingeschränkt möglich und es konnten mehrheitlich nur Tendenzen aufgezeigt werden. 